Discrete Comput Geom 35:513-523 (2006)

DOI: $10.1007 / \mathrm{s} 00454-005-1229-4$

\title{
Tverberg-Type Theorems for Separoids
}

\author{
Juan José Montellano-Ballesteros, ${ }^{1}$ Attila Pór, ${ }^{2}$ and Ricardo Strausz ${ }^{1,2}$ \\ ${ }^{1}$ Instituto de Matemáticas, U.N.A.M., \\ Circuito Exterior, C.U., 04510 México D.F., México \\ \{juancho,strausz\}@math.unam.mx \\ ${ }^{2}$ Institut Teoretické Informatiky, Universita Karlova v Praze, \\ Malostranské nám. 25, Praha 1, Czech Republic \\ por@kam.mff.cuni.cz
}

\begin{abstract}
Let $S$ be a $d$-dimensional separoid of $(k-1)(d+1)+1$ convex sets in some "large-dimensional" Euclidean space $\mathbb{E}^{N}$. We prove a theorem that can be interpreted as follows: if the separoid $S$ can be mapped with a monomorphism to a d-dimensional separoid of points $P$ in general position, then there exists a $k$-colouring $\varsigma: S \rightarrow K_{k}$ such that, for each pair of colours $i, j \in K_{k}$, the convex hulls of their preimages do intersect-they are not separated. Here, by a monomorphism we mean an injective function such that the preimage of separated sets are separated. In a sense, this result is "dual" to the Hadwiger-type theorems proved by Goodman and Pollack (1988) and Arocha et al. (2002).

We also introduce $\vartheta(k, d)$, the minimum number $n$ such that all $d$-dimensional separoids of order at least $n$ can be $k$-coloured as before. By means of examples and explicit colourings,
\end{abstract} we show that for all $k>2$ and $d>0$,

$$
(k-1)(d+1)+1<\vartheta(k, d)<\left(\begin{array}{l}
k \\
2
\end{array}\right)(d+1)+1 .
$$

Furthermore, by means of a probabilistic argument, we show that for each $d$ there exists a constant $C=C(d)$ such that for all $k, \vartheta(k, d) \leq C k \log k$.

\section{Introduction and Statement of Results}

As suggested by Danzer et al. (1963) [4], the relationship between Helly's, Radon's and Carathéodory's theorems "could be best understood by formulating various axiomatic settings for the theory of convexity". The first attempt to give such an axiomatic setting was made by Levi (1951) [11], who uses Helly's theorem (1923) [10] as a starting point. More recently, the concept of a separoid was introduced [1], [3], [12], [13], [17]-[19] as a new attempt in this direction that is instead based on Radon's theorem (1921) [15]. 
A separoid is a (finite) set $S$ endowed with a symmetric relation $\dagger \subset\left(\begin{array}{c}2^{S} \\ 2\end{array}\right)$ defined on its family of subsets, which satisfies the following properties for all $A, B \subseteq S$ :

(i) $A \dagger B \Longrightarrow A \cap B=\emptyset$,

(ii) $A \dagger B$ and $B \subset B^{\prime}(\subseteq S \backslash A) \Longrightarrow A \dagger B^{\prime}$.

A pair $A \dagger B$ is called a Radon partition. Each part ( $A$ and $B$ ) is called a component and the union $A \cup B$ is the support of the partition. The (combinatorial) dimension of $S$, denoted by $\mathrm{d}(S)$, is the minimum $d$ such that every subset of $S$ with at least $d+2$ elements is the support of a Radon partition. By the second condition, the minimal Radon partitions determine the separoid. A pair of disjoint subsets $\alpha, \beta \subseteq S$ that is not a Radon partition is said to be separated, and denoted by $\alpha \mid \beta$ (see [1]).

Now, given a family of convex sets $\mathcal{F}=\left\{C_{1}, \ldots, C_{n}\right\}$ in some Euclidean space $\mathbb{E}^{d}$, a separoid $S(\mathcal{F})$ on $\{1, \ldots, n\}$ can be defined by the following relation: for all $\alpha, \beta \subseteq S(\mathcal{F})$,

$$
\alpha \mid \beta \Longleftrightarrow\left\langle\bigcup_{i \in \alpha} C_{i}\right\rangle \cap\left\langle\bigcup_{j \in \beta} C_{j}\right\rangle=\emptyset,
$$

where $\langle\cdot\rangle$ denotes the convex hull. Analogously, the Radon partitions are defined by

$$
A \dagger B \quad \Longleftrightarrow \quad A \cap B=\emptyset \quad \text { and } \quad\left\langle\bigcup_{i \in A} C_{i}\right\rangle \cap\left\langle\bigcup_{j \in B} C_{j}\right\rangle \neq \emptyset .
$$

Conversely, as proved by Arocha et al. [1], every (abstract) separoid can be represented in such a way by a family of convex sets in some Euclidean space. Therefore each separoid $S$ has a minimum dimension where it can be represented called the geometric dimension of $S$, denoted by $\operatorname{gd}(S)$. Furthermore, as proved by Strausz [19], if the separoid $S$ is acyclic (i.e., if $\emptyset \mid S$ ), then $\operatorname{gd}(S) \leq|S|-1$ (see also [17]).

The following theorem is an easy corollary of Tverberg's theorem [20] (see also [5] and the references therein).

Theorem 1. Let $S$ be a separoid of order $|S|=(k-1)(d+1)+1$, where $d=\operatorname{gd}(S)$. Then there exists a $k$-colouring $\varsigma: S \rightarrow K_{k}=\{1, \ldots, k\}$ such that every pair of chromatic classes is not separated; i.e., the preimage of every pair of colours $i, j \in K_{k}$, is a Radon partition $\varsigma^{-1}(i) \dagger \varsigma^{-1}(j)$.

Indeed, a stronger conclusion can be reached. Represent the separoid $S$ with convex sets in $\mathbb{E}^{d}$, where $d=\operatorname{gd}(S)$. If we choose a point in each convex set and apply Tverberg's theorem to this set of points, then we can find a $k$-colouring of $S$ such that there is a point that is in the convex hull of every chromatic class.

Following [18], if the conclusion of Theorem 1 holds, we say that there exists a chromomorphism onto the complete separoid $K_{k}$ of order $k$ (to be defined; see Fig. 1). If such a chromomorphism exists for a given $S$, we write $S \longrightarrow K_{k}$; otherwise we write $S \nrightarrow K_{k}$. In this note we are interested in purely combinatorial conditions that guarantee the existence of chromomorphisms onto complete separoids.

As shown by Fig. 2, in Theorem 1 the geometric dimension cannot be replaced by the combinatorial dimension without adding a new ingredient-observe that $\mathrm{d}(S) \leq \operatorname{gd}(S)$. 


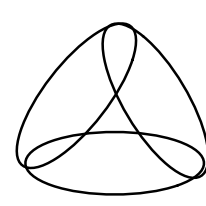

(a)



(b)

Fig. 1. Two representations of $K_{3}$ in $\mathbb{E}^{2}$.

Thus, while replacing $\operatorname{gd}(S)$ by $\mathrm{d}(S)$, we may add a Hadwiger-type hypothesis that allows us to prove the following:

Theorem 2. Let $S$ be a d-dimensional separoid of order $|S|=(k-1)(d+1)+1$. Suppose that in addition, there exists a d-dimensional separoid of points $P$ in general position and a monomorphism from $S$ into $P$ (i.e., an injective function $\mu: S \rightarrow P$ such that the preimage of separated sets are separated). Then $S \longrightarrow K_{k}$.

Arocha et al. [1] proved a Hadwiger-type theorem that, supposing the existence of a monomorphism "from the left" $v: P \rightarrow S$, concludes the existence of a virtual $\ell$ transversal. That is, there are "as many" hyperplanes transversal to the family as there are hyperplanes through an $\ell$-flat (e.g., while the family in Fig. 1(b) has a 0-transversal, that of Fig. 1(a) has a virtual 0 -transversal). This result extends ideas from Goodman and Pollack [7] who used the notion of order type to characterise the existence of hyperplane transversals. On the other hand, Theorem 2 supposes the existence of a monomorphism "to the right", and concludes that there is a virtual Tverberg partition (i.e., a partition with a virtual 0-transversal). Thus these theorems may be seen as "dual"- at least in the case $\ell=\mathrm{d}(S)$.

However, the Hadwiger-type hypotheses are "geometric" in nature; that is, they restrict the convex sets that represent the separoid to be in some "special position". (See [4] for the early work on such "special position" hypotheses, and see [5] and [8] for excellent updates on the subject.) The following questions arise. How far can the hypothesis of Theorem 2 be weakened without changing the conclusion? Is there a purely combinatorial Tverberg-type theorem?



Fig. 2. A one-dimensional separoid $S$ of five convex sets in $\mathbb{E}^{2}$ such that $S \nrightarrow K_{3}$. 
We now introduce the following new concept. The $(k, d)$-Tverberg number $\vartheta(k, d)$ is the minimum number $n \in \mathbb{N}$ such that every $d$-dimensional separoid of order at least $n$ maps onto $K_{k}$ with a chromomorphism; that is, $\vartheta(k, d)$ is minimal with the property

$$
|S| \geq \vartheta(k, \mathrm{~d}(S)) \quad \Longrightarrow \quad S \longrightarrow K_{k} .
$$

Analogously, if $\mathcal{S}$ denotes a class of separoids, we denote by $\vartheta_{\mathcal{S}}(k, d)$ the $(k, d)$-Tverberg number restricted to the class $\mathcal{S}$. Thus, Tverberg's theorem can be rewriten as

$$
\vartheta_{\mathcal{P}}(k, d)=(k-1)(d+1)+1,
$$

where $\mathcal{P}$ denotes the class of separoids of points. Analogously, using the notion of pseudoconfiguration of points, Roudneff [16] proved that

$$
\vartheta_{\mathcal{M}}(k, 2)=3 k-2,
$$

where $\mathcal{M}$ denotes the class of oriented matroids. In this direction we prove the following:

Proposition 1. If $\mathcal{G}$ denotes the class of (simple) graphs—thought of as separoids whose minimal Radon partitions are pairs of singletons - then

$$
\vartheta_{\mathcal{G}}(k, d) \leq(k-1)(d+1)+1 .
$$

(Observe the close relation between $\vartheta_{\mathcal{G}}(k, d)$ and the so-called pseudoachromatic number [9].)

However, in general the $(k, d)$-Tverberg number is greater than that. Indeed, we will prove that

Theorem 3. For all pairs of natural numbers $k>2$ and $d>0$ it follows that

$$
(k-1)(d+1)+1<\vartheta(k, d)<\left(\begin{array}{l}
k \\
2
\end{array}\right)(d+1)+1 .
$$

Furthermore, by means of a probabilistic argument, we will prove that

Theorem 4. For each $d>0$, the constant $C=2^{d+4}$ is such that for all $k \geq d+2$,

$$
\vartheta(k, d) \leq C k \log k
$$

\section{Definitions and Proof of Theorem 2}

In order to be self-contained, we start with some basic notions and examples. Every (finite and acyclic) separoid $S$ can be represented by a family of (convex) polytopes in the $(|S|-1)$-dimensional Euclidian space [17], [19]. The construction is as follows. Let $S$ be identified with the set $\{1, \ldots, n\}$. For each element $i \in S$ and each minimal Radon partition $A \dagger B$ such that $i \in A$, consider the point

$$
\rho_{A \dagger B}^{i}=\mathbf{e}_{i}+\frac{1}{2}\left[\frac{1}{|B|} \sum_{b \in B} \mathbf{e}_{b}-\frac{1}{|A|} \sum_{a \in A} \mathbf{e}_{a}\right],
$$


where $\mathbf{e}_{i}$ denotes the $i$ th vector of the canonical basis of $\mathbb{R}^{n}$. Then each element $i$ is represented by the convex hull of all such elements:

$$
i \mapsto \mathcal{K}_{i}=\left\langle\rho_{A \dagger B}^{i}: i \in A \text { and } A \dagger B\right\rangle .
$$

Observe that the convex sets $\mathcal{K}_{i}$ live in the affine hyperplane spanned by the basis. It is simple to verify that this construction is correct and that the implicit bound $n-1$ is tight.

Thus there is a minimum dimension in which $S$ can be represented, called the geometric dimension of $S$ and denoted by $\operatorname{gd}(S)$. Furthermore, if the separoid can be represented by a family of points in some Euclidian space, it is called a point separoid [3], [12] (also known as a linear oriented matroid [2] or as an order type [6]).

The order of the separoid $S$ is the cardinal $|S|$ and its size is the cardinal $|\dagger|$ (i.e., the number of Radon partitions). The separoid of order $d+1$ and size 0 is called the $d$-dimensional simploid; that is, a separoid is a simploid if every subset is separated from its complement. Simploids can be represented by the vertex sets of simplices-hence the name. The (combinatorial) dimension of a separoid $S$ is the maximum dimension of its induced simploids and is denoted by $\mathrm{d}(S)$ (observe that this definition is equivalent to that given in the Introduction).

We say that the separoid is in general position if every set of $\mathrm{d}(S)+1$ elements induces a simploid. Thus, a $d$-dimensional separoid of convex sets is in general position if (and only if) every $d+2$ elements admit a $d$-flat transversal but no $d+1$ elements do. Furthermore, $d$ is the minimum number with that property.

A separoid is called a Radon separoid if each minimal Radon partition is unique in its support; i.e., if $A \dagger B, C \dagger D$ are minimal then

$$
A \cup B \subseteq C \cup D \quad \Longrightarrow \quad\{A, B\}=\{C, D\} .
$$

Observe that if $S$ is a point separoid, then $\mathrm{d}(S)=\operatorname{gd}(S)$ and it is a Radon separoid. Furthermore, a separoid $S$ in general position is a point separoid if and only if d $(S)=$ $\operatorname{gd}(S)$ (see [3]).

The (acyclic) separoid $K$ is complete if for all $i, j \in K$ we have that $i \dagger j$; i.e., if its size is as big as possible. We denote by $K_{k}$ the complete separoid of order $k$. Observe that a separoid is complete if and only if its dimension is zero.

Given two separoids $S$ and $P$, a function $\varphi: S \rightarrow P$ is a morphism if the preimage of separations are separations (see [1] and [17] for several important examples of morphisms); that is, for all $\alpha, \beta \subseteq P$,

$$
\alpha\left|\beta \quad \Longrightarrow \quad \varphi^{-1}(\alpha)\right| \varphi^{-1}(\beta) .
$$

If the function $\varphi$ is injective (resp. surjective), the morphism is called a monomorphism (resp. an epimorphism). An epimorphism is a chromomorphism if the preimage of minimal Radon partitions are Radon partitions.

The main example to have in mind while thinking about chromomorphisms is the following - it motivates the name of such morphisms. Consider a family of convex sets $S=\left\{C_{1}, \ldots, C_{n}\right\}$. Given an (effective) $k$-colouring $\varsigma: S \rightarrow\{1, \ldots, k\}$, let $D_{i}=$ $\left\langle\varsigma^{-1}(i)\right\rangle$ be the convex hull of the union of those convex sets coloured $i$, for $i=1, \ldots, k$. Let $T=\left\{D_{1}, \ldots, D_{k}\right\}$. The induced function, also denoted by $\varsigma: S \longrightarrow T$, is a chromomorphism between those separoids. 
Given a (simple and undirected) graph $G=(V, E)$, a separoid $S$ on $V$ can be defined with the relation, for $i, j \in V$,

$$
i \dagger j \text { is minimal } \Longleftrightarrow \quad i j \in E .
$$

Indeed, this definition induces a functoral embedding from the category of graphs into that of separoids when both classes are endowed with homomorphisms (see [13]). Conversely, given a separoid $S$, we say that $S$ is a graph if, for $A, B \subseteq S$,

$$
A \dagger B \text { is minimal } \Longrightarrow|A||B|=1 \text {. }
$$

Clearly $K_{k}$ is the complete graph of order $k$-hence the notation. Observe that a graph $H$ is a minor of a connected graph $G$ if and only if there exists a chromomorphism $G \longrightarrow H$ with all its fibres connected.

Proof of Proposition 1. Let $G$ be a $d$-dimensional graph. We need to prove that

$$
|G| \geq(k-1)(d+1)+1 \quad \Longrightarrow \quad G \longrightarrow K_{k} .
$$

Denote by $\alpha(G)=d+1$ the independence number and by $\chi(G)$ the chromatic number. Using the well-known Erdôs inequality, $|G| \leq \chi(G) \alpha(G)$, we have that

$$
(k-1) \alpha(G)+1 \leq|G|<\chi(G) \alpha(G)+1,
$$

which implies that $k \leq \chi(G)$. Observe that any homomorphism-or proper colouring if you will-

$$
\varphi: G \rightarrow K_{\chi(G)}
$$

is also a chromomorphism. Furthermore, for all $n \leq m$ there is a chromomorphism $K_{m} \longrightarrow K_{n}$. Therefore, there is a chromomorphism $\psi: K_{\chi(G)} \longrightarrow K_{k}$ and we have that $\varsigma=\psi \circ \varphi$ is the desired chromomorphism.

Proof of Theorem 2. Let $S$ be a $d$-dimensional separoid of order $(k-1)(d+1)+1$. Suppose there is a monomorphism $\mu: S \rightarrow P$ into a $d$-dimensional point separoid in general position. Due to Tverberg's theorem, there exists a chromomorphism $\tau: P \longrightarrow$ $K_{k}$. We now show that $\varsigma=\tau \circ \mu$ is a chromomorphism.

Let $i \dagger j$ be an edge of $K_{k}$. Since $\tau$ is a chromomorphism, we have that $\tau^{-1}(i) \dagger \tau^{-1}(j)$. Then there exist $A \subseteq \tau^{-1}(i)$ and $B \subseteq \tau^{-1}(j)$ such that $A \dagger B$ is minimal. Since $P$ is in general position, $|A \cup B|=d+2$. Since $\mu$ is injective, $\left|\mu^{-1}(A \cup B)\right|=d+2$ and there exist $C \dagger D$ such that $C \cup D=\mu^{-1}(A \cup B)$. Therefore, since $\mu$ is a monomorphism, $\mu(C) \dagger \mu(D)$. Since $P$ is a point separoid, it is a Radon separoid and we may suppose that $\mu(C)=A$ and $\mu(D)=B$. Finally, since $C \subseteq \varsigma^{-1}(i)$ and $D \subseteq \varsigma^{-1}(j)$, we have that $\varsigma^{-1}(i) \dagger \varsigma^{-1}(j)$, which concludes the proof.

Figure 3 shows that the hypothesis of general position cannot be dropped without adding a new ingredient. On the other hand, if we suppose-as did Goodman and Pollack [7] and Arocha et al. [1] - that the monomorphism comes "from the left" $\mu: P \rightarrow S$, then such a hypothesis is not needed and the argument is much simpler (see the proof of 


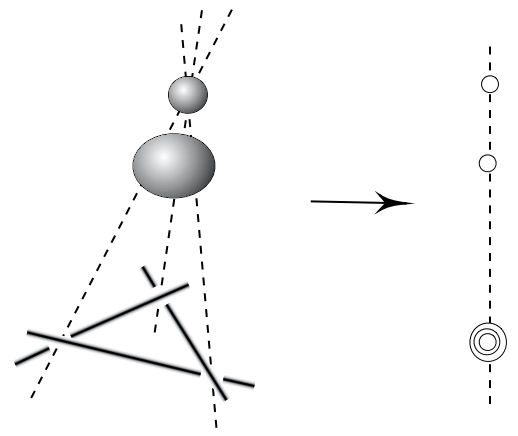

Fig. 3. A one-dimensional separoid $S$ of five convex sets in $\mathbb{E}^{3}$ such that $S \nrightarrow K_{3}$.

Lemma 1). Observe that Fig. 3 also shows that the existence of a virtual line does not imply the existence of the corresponding chromomorphism.

\section{Proofs of Theorems 3 and 4}

We start this section with a simple, but useful, structural result that allows us to restrict our attention to Radon separoids in general position.

Lemma 1. Given a d-dimensional separoid $S$, there exists a d-dimensional Radon separoid $R$ in general position such that

$$
R \longrightarrow K_{k} \quad \Longrightarrow \quad S \longrightarrow K_{k}
$$

Proof. Let $R$ be defined on the same set as $S$, and with the following set of minimal Radon partitions: for each subset $X \in\left(\begin{array}{c}S \\ d+2\end{array}\right)$, choose a single Radon partition $A \dagger(X \backslash A)$ of $S$ to be in $R$. Clearly $R$ is a Radon separoid in general position and $\mathrm{d}(R)=\mathrm{d}(S)$. Furthermore, the identity map $v: R \rightarrow S$ is a monomorphism.

Now, suppose that $\varsigma: R \longrightarrow K_{k}$ is a chromomorphism; that is, suppose that for each edge $i \dagger j$ of $K_{k}$ it follows that $\varsigma^{-1}(i) \dagger \varsigma^{-1}(j)$. Since $v$ is a monomorphism, we have that $v \circ \varsigma^{-1}(i) \dagger v \circ \varsigma^{-1}(j)$. Therefore, $\zeta \circ v^{-1}: S \longrightarrow K_{k}$ is a chromomorphism which concludes the proof.

In what follows, given a partition $X_{1}, \ldots, X_{k}$ we say that it has type $\left(\left|X_{1}\right|, \ldots,\left|X_{k}\right|\right)$. In particular, given a Radon partition $A \dagger B$ we denote its type as the pair $(|A|,|B|)$.

Theorem 3 follows immediately from the following two lemmas.

Lemma 2. For all $k>2$ and $d>0$ there exists a d-dimensional separoid $S$ of order

$$
|S|=(k-1)(d+1)+1
$$

such that $S \nrightarrow K_{k}$. 
Proof. Let $S=\{0,1, \ldots,(k-1)(d+1)\}$ be endowed with the following minimal Radon partitions: for each $A \in\left(\begin{array}{c}S \\ d+2\end{array}\right)$ let $x \dagger(A \backslash x)$, where $x \in A$ is chosen such that

$$
x=\left\{\begin{array}{lll}
0 & \text { if } 0,1 \in A, \\
1 & \text { if } 0 \notin A \text { and } 1 \in A, \\
a \neq 0 & \text { if } 0 \in A \text { and } 1 \notin A, \\
a & \text { if } 0,1 \notin A .
\end{array}\right.
$$

Since each minimal Radon partition has type $(1, d+1)$, if $S \longrightarrow K_{k}$ then the induced partition must have type $(1, d+1, \ldots, d+1)$. That isolated element in the partition is called the singleton.

Now, suppose that $S \longrightarrow K_{k}$ and look at the partition induced by such a colouring. The singleton cannot be 0 because $0 \dagger(A \backslash 0)$ only if $1 \in A$, and 1 can only be in one part. The singleton cannot be 1 because $1 \dagger(A \backslash 1)$ only if $0 \notin A$ and 0 must be in some part. Thus suppose $a \notin\{0,1\}$ is the singleton. However, then $a \mid \llbracket 1 \rrbracket$, where $\llbracket 1 \rrbracket$ denotes the chromatic class of 1 ; this is a contradiction.

Lemma 3. Let $S$ be a d-dimensional separoid. Then

$$
|S|=\left(\begin{array}{l}
k \\
2
\end{array}\right)(d+1) \quad \Longrightarrow \quad S \longrightarrow K_{k} .
$$

Proof. Let $H=H_{k}$ denote the graph resulting from deleting an edge to $K_{k}$; that is, $H$ is the set (of colours) $\{1, \ldots, k\}$ and for each pair $i j \in\left(\begin{array}{l}k \\ 2\end{array}\right)$ except for one, say $1 k$, there is an edge $i \dagger j$. Let

$$
S=\bigcup_{i j \in\left(\begin{array}{l}
k \\
2
\end{array}\right)} S_{i j}
$$

be a partition of type $(d+2, d+1, \ldots, d+1, d)$. Furthermore, suppose that $\left|S_{12}\right|=d+2$ and $\left|S_{1 k}\right|=d$. Below we exhibit a chromomorphism $\varsigma: S \backslash S_{1 k} \longrightarrow H$. Then we extend it to a chromomorphism onto $K_{k}$ using the remaining $d$ elements of $S$.

Since $\left|S_{12}\right|=d+2$, there is a Radon partition $A \dagger B$ whose support is $S_{12}$. Assign colours respectively (i.e., let $\varsigma(A)=1$ and $\varsigma(B)=2$ ).

Remark. We may suppose that $A$ has the maximum size that a component may have in $S \backslash S_{1 k}$.

Choose any element of colour 1 , say $a \in A$. We now use $a$ to extend the colouring to the parts $S_{1 j}$ (with $1<j \neq k$ ) so that $\varsigma$ becomes onto the edges incident to 1 . That is, for each pair $1 j \in\left(\begin{array}{l}k \\ 2\end{array}\right) \backslash 1 k$, the set $S_{1 j} \cup a$, which consists of $d+2$ elements, defines a Radon partition $A^{\prime} \dagger B^{\prime}$ (we may suppose $a \in A^{\prime}$ ). Thus, we can assign colours by $\varsigma\left(A^{\prime}\right)=1$ and $\varsigma\left(B^{\prime}\right)=j$.

Now, choose an element coloured 2, say $b \in \varsigma^{-1}(2)$, and use it to extend all parts $S_{2 j}$, with $2<j$ (i.e., consider $S_{2 j} \cup b$, take its Radon partition and assign colours). Then repeat for colour 3 , colour 4 and so on. At the end of such a process the colouring $\varsigma$ is the desired chromomorphism.

Trying to extend $\varsigma$ to a chromomorphism onto $K_{k}$, we meet one possible obstruction: if we choose two elements coloured 1 , say $a, b \in \varsigma^{-1}(1)$, and consider the set $S_{1 k} \cup\{a, b\}$ 
of $d+2$ elements, then the defined Radon partition $A^{\prime} \dagger B^{\prime}$ is such that $a \in A^{\prime}$ and $b \in B^{\prime}$ (analogously if both elements are coloured $k$ ). Also, if we choose one element of each colour, say $a \in \varsigma^{-1}(1)$ and $b \in \varsigma^{-1}(k)$, and consider $S_{1 k} \cup\{a, b\}$, then the respective Radon partition $A^{\prime} \dagger B^{\prime}$ contains both elements on the same component, say $\{a, b\} \subset A^{\prime}$.

However, by the Remark, we may suppose that $\left|\varsigma^{-1}(1) \cup \varsigma^{-1}(k)\right| \geq d+2$. Then there is a minimal Radon partition $C \dagger D$ whose support is contained in the preimage of this "missing edge". Furthermore, since $\varsigma^{-1}(1) \mid \varsigma^{-1}(k)$, there exist a pair of elements $a \in \varsigma^{-1}(1)$ and $b \in \varsigma^{-1}(k)$ which appears on the same side of that Radon partition, say $\{a, b\} \subset C$. Therefore, we can apply the previous method, but starting with $S_{12}=C \cup D$, to find another chromomorphism $\varsigma^{\prime}: S \backslash S_{1 k} \longrightarrow H$ such that $\varsigma^{\prime}(C)=1$. Then $\varsigma^{\prime}$ can be extended to a chromomorphism $S \longrightarrow K_{k}$.

Given a separoid $S$ of order $2^{t} k$, we denote by $\Omega$ the set of all $k$-colourings of $S$ such that each chromatic class consists of exactly $2^{t}$ elements. Analogously, given a Radon separoid $T$ of order $2^{t+1}$ in general position we denote by $\widehat{\Omega}$ the set of all 2-partitions of $T$ into two sets of order $2^{t}$. Furthermore, a pair $(\alpha, \beta) \in \widehat{\Omega}$ is called a halving of $T$. We denote by $\mathbf{p}_{T}$ the probability that $\alpha \mid \beta$, for a randomly and uniformly choosen $(\alpha, \beta) \in \widehat{\Omega}$.

The proof of Theorem 4 is mainly based on the following:

Lemma 4. If $T$ is a d-dimensional Radon separoid of order $2^{t+1}$ in general position, then

$$
\mathbf{p}_{T} \leq(d+2) / 2^{2^{t-d-1}}
$$

Before proving this lemma, we see how it is used.

Proof of Theorem 4. Let $t=d+4+\lfloor\log \log k\rfloor$ and let $S$ be a $d$-dimensional Radon separoid of order $2^{t} k$. Since $2^{t} \leq 2^{d+4} \log k$ it is enough to prove that $S \longrightarrow K_{k}$.

Let $S=S_{1} \cup \cdots \cup S_{k}$ be a random partition of $S$. Let $E_{i, j} \subset \Omega$ be the event that $S_{i} \mid S_{j}$. We claim that

$$
\Omega \backslash \bigcup_{i j \in\left(\begin{array}{l}
k \\
2
\end{array}\right)} E_{i, j} \neq \varnothing .
$$

It is clear that all events $E_{i, j}$ have the same probability. Now, we can obtain $S_{1}$ and $S_{2}$ as follows. Randomly choose a set $T \subset S$ of order $2^{t+1}$ and let $\left(S_{1}, S_{2}\right) \in \widehat{\Omega}$ be a random halving of T. By Lemma 4 , the probability that $S_{1} \mid S_{2}$ is at most $(d+2) / 2^{2^{t-d-1}}$. Therefore

$$
\begin{aligned}
\operatorname{Prob}\left[\bigcup_{i j \in\left(\begin{array}{l}
k \\
2
\end{array}\right)} E_{i, j}\right] & \leq \sum_{i j \in\left(\begin{array}{l}
k \\
2
\end{array}\right)} \operatorname{Prob}\left[E_{i, j}\right]=\left(\begin{array}{l}
k \\
2
\end{array}\right) \operatorname{Prob}\left[E_{1,2}\right] \\
& \leq \frac{k^{2}}{2}(d+2) 2^{-2^{t-d-1}} \leq \frac{k^{2}}{2}(d+2) 2^{-2^{2+\log \log k}} \\
& =\frac{k^{2}}{2}(d+2) k^{-4}<1 .
\end{aligned}
$$


Proof of Lemma 4.. We prove the lemma by induction on $d$. If $d=-1$ and $t \geq 0$ then every element of $T$ is not separated from the empty set. Thus $\mathbf{p}_{T}=0$. Now, let $d \geq 0$ and suppose the lemma is true for all $d^{\prime}<d$. If $t \leq d$ we have that $(d+2) / 2^{2^{t-d-1}}>1$; thus let $t>d$.

We can achieve a random halving of $T$ as follows: in the first step, halve the set $T$ into two equal parts $T_{1}$ and $T_{2}$; later, randomly halve each $T_{i}(i=1,2)$ into two equal sets $\alpha_{i}$ and $\beta_{i}$. Let $\alpha=\alpha_{1} \cup \alpha_{2}$ and $\beta=\beta_{1} \cup \beta_{2}$. The halving $(\alpha, \beta) \in \widehat{\Omega}$ is random (and uniform).

We define a $(d-1)$-dimensional separoid on $T_{1}$ as follows (we use $\|$ and $\ddagger$ to denote the separations and Radon partitions on $T_{1}$, respectively). For each $X \in\left(\begin{array}{c}T_{1} \\ d+1\end{array}\right)$ and each $A \subset X$, let

$$
T(X, A)=\left\{x \in T_{2}:(A \cup x) \dagger(X \backslash A)\right\} .
$$

Observe that for two different subsets $A, A^{\prime} \subset X$ we have $T(X, A) \cap T\left(X, A^{\prime}\right)=\emptyset$. Furthermore, since for each element $x \in T_{2}$ there is a Radon partition of the set $X \cup x$, the sets $T(X, A)$, with $A \in 2^{X}$, are a partition of $T_{2}$. Thus, for some $A_{X} \subset X$ we have that $\left|T\left(X, A_{X}\right)\right| \geq\left|T_{2}\right| /\left|2^{X}\right|=2^{t-d-1}$. We define $\ddagger$ as the relation $A_{X} \ddagger\left(X \backslash A_{X}\right)$, for all subsets $X \subset T_{1}$ of order $d+1$. Observe that $\ddagger$ defines a $(d-1)$-dimensional Radon separoid in general position on $T_{1}$.

Let $E$ be the event that $\alpha \mid \beta$ and let $E_{1}$ be the event that $\alpha_{1} \mid \beta_{1}$. By the induction hypothesis we have that $\operatorname{Prob}\left[E_{1}\right] \leq(d+1) / 2^{2^{(t-1)-(d-1)-1}}=(d+1) / 2^{2^{t-d-1}}$.

Take the halving $(\alpha, \beta)$ in $E \backslash E_{1}$. Since $\alpha_{1} \ddagger \beta_{1}$, there exists $X \subset T_{1}$ of order $d+1$ such that $\left(X \cap \alpha_{1}\right) \ddagger\left(X \cap \beta_{1}\right)$. We may assume that $A_{X}=X \cap \alpha_{1}$.

We claim that $T\left(X, A_{X}\right) \cap \alpha_{2}=\emptyset$. Suppose that there exists an $x \in T\left(X, A_{X}\right) \cap \alpha_{2}$. Since $\left(A_{X} \cup x\right) \dagger\left(X \backslash A_{X}\right), A_{X} \cup x \subset \alpha$ and $X \backslash A_{X} \subset \beta$, we have that $\alpha \dagger \beta$; this is a contradiction and the claim follows.

Now, let $s=2^{t-d-1} \leq\left|T\left(X, A_{X}\right)\right|$ and let $\bar{E}_{1}=\widehat{\Omega} \backslash E_{1}$. In order for $\alpha \mid \beta$, the set $T\left(X, A_{X}\right)$ has to be contained in $\beta_{2}$. Thus the conditional probability is

$$
\frac{\operatorname{Prob}\left[E \cap \bar{E}_{1}\right]}{\operatorname{Prob}\left[\bar{E}_{1}\right]}=\operatorname{Prob}\left[E \mid \bar{E}_{1}\right] \leq \frac{\left(\begin{array}{c}
2^{t}-s \\
2^{-1}-s
\end{array}\right)}{\left(\begin{array}{c}
2^{t} \\
2^{t-1}
\end{array}\right)} \leq 2^{-s}=2^{-2^{t-d-1}} .
$$

Therefore,

$$
\begin{aligned}
\mathbf{p}_{T} & =\operatorname{Prob}[E] \leq \operatorname{Prob}\left[E_{1}\right]+\operatorname{Prob}\left[E \backslash E_{1}\right] \\
& \leq \operatorname{Prob}\left[E_{1}\right]+\frac{\operatorname{Prob}\left[E \backslash E_{1}\right]}{\operatorname{Prob}\left[\bar{E}_{1}\right]} \leq(d+2) / 2^{2^{t-d-1}} .
\end{aligned}
$$

Observe the relation with Rado's original bound on the "Tverberg's number" [14]:

$$
\vartheta_{\mathcal{P}} \leq(k-2) 2^{d}+d+2 .
$$

\section{References}

1. J.L. Arocha, J. Bracho, L. Montejano, D. Olivero and R. Strausz. Separoids: their categories and a Hadwiger-type theorem for transversals. Discrete Comput. Geom., 27 (2002), 377-385.

2. A. Björner, M. Las Vergnas, B. Sturmfels, N. White and G.M. Ziegler. Oriented Matroids. Encyclopedia of Mathematics and its Applications, vol. 43. Cambridge University Press, Cambridge, 1993. 
3. J. Bracho and R. Strausz. Separoids and a Characterization of Linear Uniform Oriented Matroids. KAMDIMATIA Series, vol. 566. Charles University, Praha, 2002.

4. L. Danzer, B. Grünbaum and V. Klee. Helly's theorem and its relatives. In Convexity (V. Klee, ed.), pp. 101-180. Proceedings of Symposia in Pure Mathematics, vol. VII, American Mathematical Society, Providence, RI, 1963.

5. J. Eckhoff. Helly, Radon and Carathéodory type theorems. In Handbook of Convex Geometry (P.M. Gruber and J. Willis, eds.), pp. 389-448. North-Holland, Amsterdam, 1993.

6. J.E. Goodman and R. Pollack. Multidimentional sorting. SIAM J. Comput., 12 (1983), 484-503.

7. J.E. Goodman and R. Pollack. Hadwiger's transversal theorem in higher dimensions. J. Amer. Math. Soc., 1 (1988), 301-309.

8. J.E. Goodman, R. Pollack and R. Wenger. Geometric transversal theory. In New Trends in Discrete and Computational Geometry (J. Pach, ed.), pp. 163-198. Springer-Verlag, New York, 1993.

9. R.P. Gupta. Bounds on the chromatic and achromatic numbers of complementary graphs. In Recent Progress in Combinatorics (W.T. Tutte, ed.), pp. 229-235. Academic Press, New York, 1969.

10. E. Helly. Über Mengen konvexer Körper mit gemeinschaftlichen Punkten. Jahresber. Deutsch. Math.Verein., 32 (1923), 175-176.

11. F.W. Levi. On Helly's theorem and the axioms of convexity. J. Indian Math. Soc. (N.S.) Part A, 15 (1951), 65-76.

12. J.J. Montellano-Ballesteros and R. Strausz. Counting polytopes via the Radon complex. J. Combin. Theory Ser. A, 106 (2004), 109-121.

13. J. Nešetřil and R. Strausz. Universality of separoids. Arch. Math. (Brno) (2005).

14. R. Rado. Theorems of intersection of convex sets of points. J. London Math. Soc., 27 (1952), 320-328

15. J. Radon. Mengen konvexer Körper, die einen gemeinsamen Punkt enthalten. Math. Ann., 83 (1921), 113-115.

16. J.-P. Roudneff. Tverberg-type theorems for pseudoconfigurations of points in the plane. European $J$. Combin., 9 (1988), 189-198.

17. R. Strausz. On Radon's Theorem and Representation of Separoids. ITI-Series, vol. 118. Charles University, Praha, 2003.

18. R. Strausz. Representation of separoids and a Tverberg-type problem. In Proc. IXth Midsummer Combinatorial Workshop, Prague, 2002 (M. Mareš and J. Nešetřil, eds.), pp. 42-51. KAM-DIMATIA Series, vol. 689. Charles University, Praha, 2004.

19. R. Strausz. On Separoids. Ph.D. Thesis, Universidad Nacional Autónoma de México, 2004.

20. H. Tverberg. A generalization of Radon's theorem. J. London Math. Soc., 41 (1966), 123-128.

Received October 29, 2004, and in revised form October 18, 2005. Online publication February 3, 2006. 